\title{
DESIGN AND EVALUATION OF SERIAL-HYBRID VEHICLE ENERGY GAUGES
}

\author{
Janet Creaser $^{1}$, John Lenneman ${ }^{2}$, Joseph Szczerba ${ }^{2}$ \\ ${ }^{1}$ HumanFIRST Program, ITS Institute \\ University of Minnesota \\ Minneapolis, MN, USA \\ ${ }^{2}$ General Motors R\&D and Planning \\ Warren, MI, USA
}

Email: janetc@me.umn.edu; john.lenneman@gm.com; joe.szczerba@gm.com

\begin{abstract}
Summary: This paper describes a usability study of serial-hybrid vehicle energy gauge designs. Eight gauges that were modified by design format (bars, dials), color (one color, two colors) and the type of information present (range information, no range information) were tested in a driving simulator under urban/suburban traffic conditions. Participants answered questions about the state of the battery and fuel tank separately and also answered questions that involved combining the information from both sources of energy. Comprehension was assessed based on accuracy and response times to the questions when a gauge was presented. Participants also completed subjective ratings of the gauges. Driving performance was assessed to determine if driving was affected by responding to gauge presentations. Overall, the results indicated that the bar design using two colors and including range information performed best when integration of the two energy sources was required. These attributes were also most preferred by participants in this study.
\end{abstract}

\section{INTRODUCTION}

Recently, serial hybrids have been announced as another alternative energy system that will be offered to the general public as early as the year 2010. A serial hybrid is a vehicle where the batteries can be recharged by connecting the vehicle to a standard electric outlet (i.e., "plug-in hybrid"). This differs from most current hybrid vehicles, known as parallel hybrids, where power is simultaneously conveyed to the wheels from two separate energy sources, such as an internal combustion engine and a battery. The new serial hybrids will have two separate energy sources that power the vehicle in series rather than in parallel. First, the stored electric energy from the battery is utilized to exclusively power the vehicle. Once this energy is depleted, the additional energy source (e.g., combustion engine) is used to power the vehicle wheels.

Therefore, a serial hybrid can operate over a considerable distance without engaging the internal combustion engine. Serial hybrids can also operate without recharging the battery, as long as there is liquid fuel in the tank.

The serial hybrid vehicle design requires additional energy information that needs to be conveyed to the driver. Currently, drivers are familiar with a single energy source (i.e., gas in the fuel tank) and the potential range of their vehicle based on that energy source alone. This is also true for parallel hybrids where only the status of the fuel tank needs to be shown. In contrast, the serial hybrid requires that both the status of the battery and the fuel tank be presented to the driver to provide a full picture of the vehicle's potential driving range. User understanding of the status of these two distinct energy sources then proves essential for proper vehicle operation. 
Because drivers are familiar with a single energy source in vehicles, this previous experience could be a source of confusion for drivers when additional energy information is added. The problem may be compounded if the two sources provide different potential ranges of travel for the vehicle (e.g., the fuel tank takes the vehicle 300 miles, while the electric battery only takes the vehicle 40 miles). The objective of the study was to identify how well drivers could obtain information from the recommended designs in a realistic driving environment.

\section{METHODS}

\section{Participants}

Twenty-four participants (13 males; 11 females) completed the study, and ranged in age from 22-68 $(M=40.38 ; S D=16.52)$. Participants all held a valid Minnesota Driver's License and reported driving at least once every week.

\section{Test Gauges}

GM designers provided four potential design images based on the recommendations from a previous phase of research. The research team evaluated these designs to determine how well they met the proposed recommendations and then modified the designs to better meet the original recommendations. Two final designs were selected that incorporated the best attributes from the top designs identified in a previous phase of research. These designs included the following characteristics that were considered important based on the results of the previous research:

1. Gauges were co-located in a side-by-side format

2. Gauges contained fill markings (i.e., tick marks)

3. Gauges contain a label for full (F) and empty (E)

4. Gauges are different sizes relative to one another (i.e., fuel tank gauge is bigger than battery gauge)

The goal was to compare the performance of a set of dial gauges to a set of bar gauges by manipulating the color (green vs. blue/green) and type of information available (i.e., range of vehicle for each fuel source) to the driver. Dials are more commonly used to display vehicle energy levels, but bars are a reasonable alternative because they are commonly used in other types of displays to indicate "high" and "low". Color was tested because different colors can enhance the individual nature of each information source (Wickens \& Carswell, 1995) when two energy sources are near each other, which may facilitate extraction of the individual energy source information. However, vehicle display designers may choose a single color for the entire display panel so it was important to compare single-color designs to dual-color designs based on these two competing design issues. To aid with information integration, the two energy gauges were located in close proximity to each other (Wickens \& Carswell, 1995) and the individual sources within each gauge set used the same encoding and formatting characteristics (e.g., using both bars or dials within a set; having needles point same direction for full/empty on both energy dials) to conform with the principle of consistency (Wickens, Gordon, \& Liu, 1998). Variations of each gauge set were developed according to these criteria (see Table 1). 
Table 1. Test gauges and descriptions

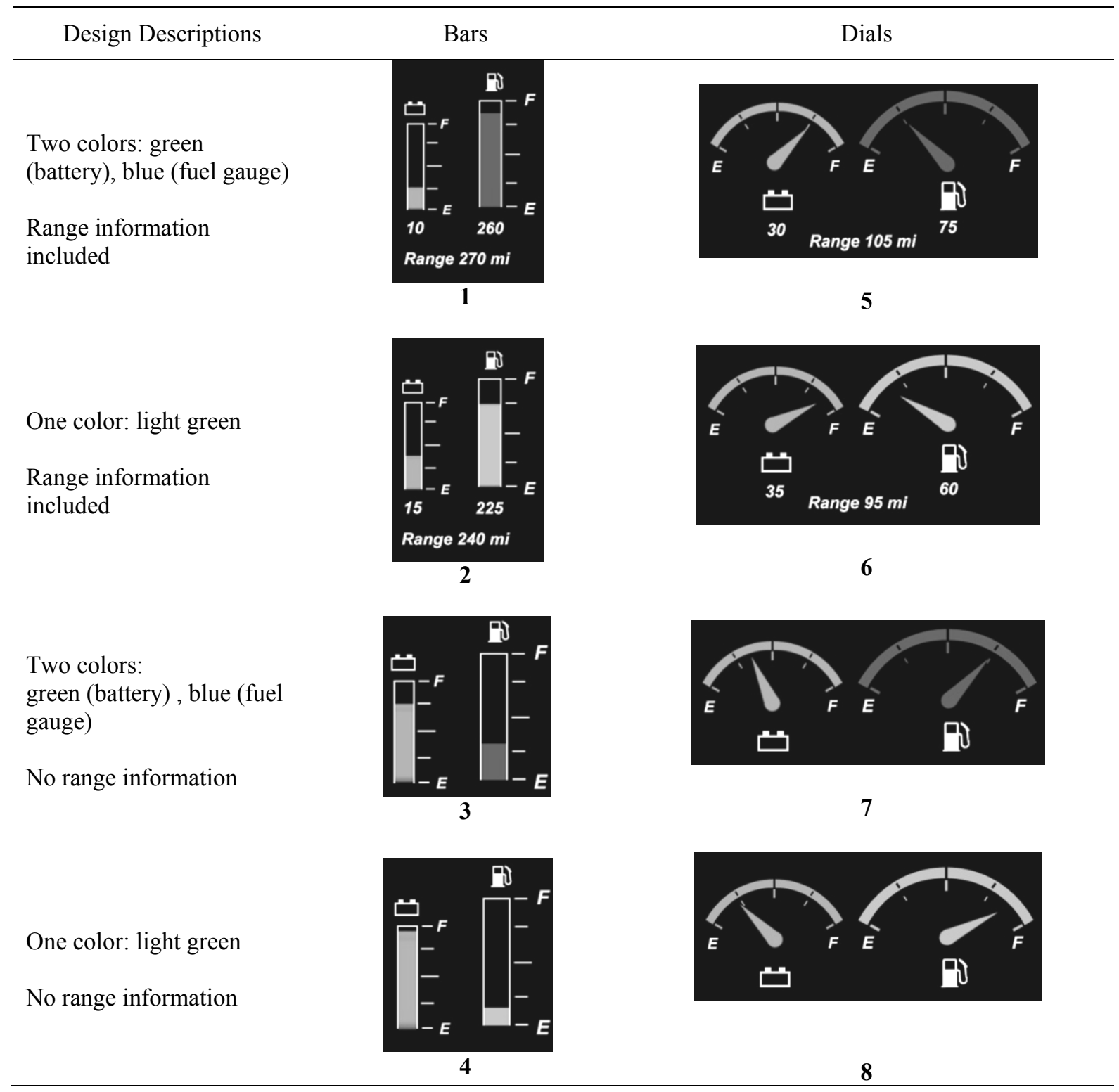

\section{Driving Simulator}

The study was conducted in the HumanFIRST Program's driving simulator (Oktal; AutoSim) located in the University of Minnesota's ITS Institute. The simulator consists of a full-sized Saturn vehicle with operational controls, a high-resolution visual scene (1.96 arc minutes per pixel) projected to a 5-channel 210-degree forward field-of-view. The rear visual scene is visible on the back screen in the vehicle's rear-view mirror. LCD panels placed on the side mirrors present a simulated side view of the driving environment. Auditory and haptic feedback are provided by a 3D surround audio system, subwoofer, car body vibration, and a three-axis electric motion system (roll, pitch, z-axis) system. For this study, an LCD panel was mounted in front of the vehicle's control panel (behind the steering wheel) for gauge presentation. Buttons on each 
side of the steering wheel were used for response inputs by participants (left button = "no"; right button $=$ "yes").

The driving environment consisted of a two-lane road with a shoulder and a sidewalk on either side and a speed limit of $45 \mathrm{mph}$. Buildings were located either next to the roadway (urban setting) or set back from the roadway (suburban setting) and cars were parked in the curb lanes. Oncoming traffic passed at a regular rate of 1-3 seconds. Moving pedestrians were located on the sidewalks on both sides. Participants followed a lead vehicle which slowly modulated its speed profile in a sinusoidal pattern throughout the drive to simulate a higher workload environment.

\section{Procedures}

Participants completed the informed consent process followed by a computerized demographic questionnaire. The study introduction included a written description of the how the hybrid energy gauges worked. Participants completed a 5-minute practice drive to familiarize them with the simulator and a second 5-minute practice drive in which they practiced driving and completing the experimental tasks. Participants were shown the LCD panel co-located with the simulator's instrument panel where the energy gauges would appear. They were informed that an auditory warning would occur in conjunction with a new gauge presentation to alert them to its presence. After the practice drives, participants completed three 10-minute experimental drives with a 5minute break in between each drive. Within each drive they saw the 8 different gauges and needed to answer "yes" or "no" to the question that applied to that drive. The following questions (one for each drive) were used to assess driver comprehension of the gauges:

1. Is it possible for me to travel 20 miles on the battery alone?

2. Is it possible for me to travel 150 miles on the gas tank alone after the battery is depleted?

3. Is it possible for me to travel 200 miles on the battery and gas tank combined? These questions probe drivers' abilities to discriminate energy levels based on their knowledge of the range capacity of each energy source. The range is 40 miles for the battery and 300 miles for the gas tank, resulting in a 340 mile range capacity when both sources are full. Participants were asked to respond as accurately and quickly as possible to each gauge presentation. Question presentation was blocked and counterbalanced across participants. The gauge presentation software randomly displayed the 8 gauges at the prescribed times during each 10-minute drive. A gauge was presented each minute starting at Minute 2 and then each minute on the minute through Minute 9 of the drive. The gauge image disappeared once participants responded using the buttons on the steering wheel.

\section{RESULTS}

\section{Q1: Is it possible for me to travel 20 miles on the battery alone?}

Overall, there was a statistically significant main effect of gauge for response time when participants answered whether the vehicle could travel 20 miles on the battery alone, $F(7,161)=2.80, p=0.009$ (see Table 2). Post-hoc comparisons showed that Gauge $4(M=2.48 \mathrm{~s}$; $S D=0.59)$ had a significantly faster response time than Gauge $7(M=3.13 \mathrm{~s} ; S D=0.84)$. Gauge 4 is the single-color bar gauge without range information while Gauge 7 is the two-color dial gauge without range information. The single-color gauges $(M=2.81 \mathrm{~s} ; S D=0.63)$ also had statistically 
significantly faster response times than the two-color gauges $(M=3.11 \mathrm{~s} ; S D=0.77), t(23)=3.03$, $p=0.006$. Gauge 1 and Gauge 7 had the highest accuracy rates at 96\%. Gauge $8(82 \%)$ and Gauge $5(83 \%)$ had the lowest accuracy rates.

Table 2. Response times (RT), accuracy rate (\% correct) and percentage of participants who preferred each gauge (\% preferred)

\begin{tabular}{lccccccc}
\hline & \multicolumn{2}{c}{ Q1: 20 miles on battery? } & \multicolumn{2}{c}{ Q2: 150 miles on gas tank? } & \multicolumn{2}{c}{ Q3: 200 miles on both? } & Preference \\
\cline { 2 - 7 } Gauge* & RT & \% Correct & RT & \% Correct & RT & \% Correct & \% Preferred \\
\hline 1 (bars) & $3.38 \mathrm{~s}$ & $96 \%$ & $2.50 \mathrm{~s}$ & $100 \%$ & $2.55 \mathrm{~s}$ & $100 \%$ & $37.5 \%$ \\
2 (bars) & $3.00 \mathrm{~s}$ & $92 \%$ & $2.79 \mathrm{~s}$ & $95.8 \%$ & $4.05 \mathrm{~s}$ & $96 \%$ & $12.5 \%$ \\
3 (bars) & $2.95 \mathrm{~s}$ & $92 \%$ & $2.79 \mathrm{~s}$ & $95.8 \%$ & $4.30 \mathrm{~s}$ & $83 \%$ & $20.83 \%$ \\
4 (bars) & $2.47 \mathrm{~s}$ & $88 \%$ & $2.85 \mathrm{~s}$ & $100 \%$ & $3.83 \mathrm{~s}$ & $88 \%$ & $4.17 \%$ \\
5 (dials) & $2.99 \mathrm{~s}$ & $83 \%$ & $2.96 \mathrm{~s}$ & $87.5 \%$ & $4.02 \mathrm{~s}$ & $88 \%$ & $20.83 \%$ \\
6 (dials) & $2.66 \mathrm{~s}$ & $88 \%$ & $2.86 \mathrm{~s}$ & $100 \%$ & $4.14 \mathrm{~s}$ & $100 \%$ & $4.17 \%$ \\
7 (dials) & $3.13 \mathrm{~s}$ & $96 \%$ & $3.28 \mathrm{~s}$ & $100 \%$ & $4.22 \mathrm{~s}$ & $92 \%$ & $0 \%$ \\
8 (dials) & $3.07 \mathrm{~s}$ & $82 \%$ & $2.52 \mathrm{~s}$ & $100 \%$ & $3.01 \mathrm{~s}$ & $96 \%$ & $0 \%$ \\
\hline
\end{tabular}

* Gauge images are in Table 1.

Q2: Is it possible for me to travel 150 miles on the gas tank alone after the battery is depleted?

Overall, there were no significant differences in response times across gauges for this question. Overall, the accuracy rates were high for the gas tank question. Gauges 1, 4, 6, 7 and 8 all had accuracy rates of $100 \%$ (see Table 2 ).

\section{Q3: Is it possible for me to travel 200 miles on the battery and gas tank combined?}

Overall, there was a statistically significant main effect of gauge for response time when participants answered whether the vehicle could travel 200 miles on the battery and gas tank combined, $F(7,161)=4.93, p<0.001$. Gauge $1(M=2.57 \mathrm{~s} ; S D=0.85)$ has significantly faster response time than Gauge $2(M=4.04 \mathrm{~s} ; S D=1.97)$, Gauge $3(M=4.30 \mathrm{~s}$; SD1.72), Gauge 5 $(M=4.05 \mathrm{~s} ; S D=1.56)$, Gauge $6(M=4.15 \mathrm{~s} ; S D=2.00)$, and Gauge $7(M=4.22 \mathrm{~s} ; S D=1.52)$. Gauge $1(100 \%)$ and Gauge $6(100 \%)$ had the highest accuracy rates for the combined questions (see Table 2$)$. Gauge 3 had the lowest rate $(83 \%)$.

Overall, the combined information question $(\mathrm{Q} 3: M=3.78 \mathrm{~s} ; S D=0.98)$ required significantly more time to respond to than the individual battery $(\mathrm{Q} 1 ; M=2.96 \mathrm{~s} ; S D=0.65)$ or gas tank $(\mathrm{Q} 2$; $M=2.82 \mathrm{~s} ; S D=0.76$ ) questions, $F(2,46)=24.05, p<0.001$. This was expected because participants needed to integrate the two sources of information to answer the question. 


\section{Subjective Preference}

Overall, participants preferred bars to dials (73.9\% vs. $26.1 \%$ ), two-color gauges to one-color gauges $(70.8 \%$ vs. $29.2 \%)$, and the presence of range information to no range information $(75 \%$ vs. $25 \%)$.

\section{Driving Performance Data}

Driving performance data was analyzed before, during and after a gauge presentation. Data was collected for $10 \mathrm{~s}$ before a gauge presentation, for the duration of the presentation until a response was detected, and $10 \mathrm{~s}$ after the participant's response occurred. Overall, there were no significant differences in performance between gauges while driving.

\section{DISCUSSION}

The goal of this study was to identify how well drivers could obtain information from the recommended design gauges while driving in a driving simulator. Overall, the objective data indicate that Gauge 1 performed well for all three questions. Particularly, it performed well for Question 3, which required participants to integrate the two types of energy information to answer the question. This gauge was also most preferred by participants. A number of factors may have influenced the performance of this gauge.

As mentioned, all eight gauges met the proximity compatibility principle and the principle of consistency (Wickens \& Carswell, 1995; Wickens et al., 1998). The differences in performance for the bar gauges in Questions 1 and 3 may be due to other design attributes that facilitate the acquisition of information. For example, the vertical nature of the bars and the increasing/decreasing color fills map well to concepts of "full" and "empty". This design attribute is consistent with the principle of the moving part described by Roscoe (1968; as cited in Wickens et al., 1998), where a moving element on a dynamic display should move in a manner compatible with the user's mental model of how that element moves in the physical system. In contrast, the dials use a right-to-left display for full-to-empty. While right-to-left is often associated with decreases in value, it can be argued that the mental model for a decrease in value is stronger in the vertical as opposed to the horizontal direction. Thus, although this horizontal layout is common for fuel gauges in vehicles, it did not perform as well overall for the integration task. Gauge 1 significantly outperformed three of the four dial designs.

The use of bars to indicate fuel levels is also a recommended ecological interface design (EID) option for vehicles (Burns \& Hadjukiewicz, 2004). Bars are not the most commonly used display format for fuel levels in a vehicle, but they demonstrated their potential advantage in this study over the more commonly used dial format. That bars have been used in an example of EID suggests that bars are a good option for depicting fuel levels, despite the familiarity of the dials. Participants also preferred the bar gauges and reported that they found it easier to compare the two bar gauges to one another than the two dials. The fill used for the bars covers more area on the gauge than the dial needles, thus providing a larger visual target when seeking the fuel level information. For example, Burns and Hadjukiewicz (2004) described how the area filled by the bar maps to the percentage full while the height of the bar shows the level. Comments from 
participants support this assumption (e.g., "I believe that the different color bars placed side by side are easier to read at a quick glance"; "Ordinarily, I would prefer dials, but to compare two readings I find bars easier").

Although different colors can enhance the individual nature of each information source (Wickens \& Carswell, 1995), this result was not seen in this study. For Question 1, the single color gauges performed better overall when participants were extracting just battery information, but this result was not seen for Question 2. In Question 3, the dual-color bar gauge with range information performed better than the single-color bar gauge with range information (i.e., equivalent gauges except for color). These results suggest that color may not impact the integration of information or the acquisition of information from single sources, and that the results may be due to combinations of attributes, such as for Gauge 1 in Question 3 where all possible manipulations of the gauge existed (e.g., two colors, range information present). In general, design principles support the use of different colors when individual information from one gauge needs to be acquired from a set of gauges (Wickens \& Carswell, 1995). Two-color gauges were preferred by participants. Although blue and green were used here, it is likely that any two colors with good contrast against the black LCD screen and that are easily discriminated from each other could be used. Additionally, the choice of colors should not conflict with colors intended to indicate low fuel (e.g., red) or a problem with the vehicle (e.g., orange).

Finally, the results showed that integration of information from two energy sources was more time-consuming than seeking out a single information source from a set of gauges. This occurred even though total range information was included on four of the eight gauges, suggesting that drivers may not be familiar with and immediately seek the total range information for this integration task. Range information for both the individual energy sources and the combined total were preferred attributes in this study.

\section{CONCLUSIONS}

The results of this study demonstrated that the application of known design principles and the use of an uncommon design format (i.e., bars) could facilitate the acquisition of information from dual-energy sources in a serial hybrid vehicle display.

\section{REFERENCES}

Burns, C.M., Hajdukiewicz, J.R. (2004). Ecological Interface Design (pp. 85-104). New York: CRC Press.

E-Prime (v. 1.1). Psychology Software Tools, Inc. http:/www.pstnet.com/products/e-prime

Wickens, C.D., Gordon, S.E., Liu, Y. (1998). An Introduction to Human Factors Engineering (pp. 223-258). New York: Addison Wesley Longman, Inc.

Wickens, C.D. \& Carswell, C.M. (1995). The proximity compatibility principle: Its psychological foundation and relevance to display design, Human Factors, 37, 473-494. 